\title{
EAl Endorsed Transactions

\section{How instability in virtual economies of mobile digital games drives and ruins profit}

\author{
P.C. Lohse ${ }^{1, *}$ \\ ${ }^{1}$ Andrássy University, 1088 Budapest, Hungary
}

\begin{abstract}
The digital gaming business has changed in the last years. Digital games are no longer just products. They developed into services. People play games not only on a stationary device, but on mobile devices, too. The change of gaming devices also had an impact on game design. Competition between mobile digital games mostly takes place at a download price of zero. Today the Freemium monetization method is the dominant monetization method. Players of digital games often have to go through a core loop by repeating the same tasks inside the game. The stability of the virtual economy inside the game affects the game's core loop. This paper discusses the role of instability in virtual economies in the context of core loops. A small instability inside a virtual economy can increase revenue of a digital game. Players tend to stay in a game because of sunk cost fallacy. If instability inside a virtual economy happens for a too long time players will quit playing and revenue will decrease.
\end{abstract}

Keywords: Freemium, mobile gaming, sunk cost fallacy, instability in virtual economies, core loops

Received on 04 June 2019, accepted on 20 July 2019, published on 20 August 2019

Copyright $(2019$ P. C. Lohse, licensed to EAI. This is an open access article distributed under the terms of the Creative Commons Attribution licence (http://creativecommons.org/licenses/by/3.0/), which permits unlimited use, distribution and reproduction in any medium so long as the original work is properly cited.

doi: 10.4108/eai.13-7-2018.162632

"Corresponding author. Email: philipp.lohse@andrassyuni.hu

\section{Introduction}

The way people play mobile digital games has changed. Mobile digital games became more popular over the last years [10]. Mobile digital games are no longer a product. They developed into services [10] [12]. Mobile digital games with the highest revenue in the apple app store, Google Play Store or others often are available for free [7]. The majority of mobile digital games are available without a price per download and make money via in-app purchases [7]. Competition between digital mobile games mostly takes place at a download price of zero. Players pay for premium features inside the games. This monetization method is called Freemium [5]. Today Freemium is the dominant monetization method for mobile digital games [6].

In July $201698.5 \%$ of the top 200 Apple App-Store applications by revenue were available for free [7]. Only $1.5 \%$ of the top 200 Apple App-Store applications by revenue had a price per download [7]. Players can download the applications for free and test the game. If they like it, they can get an advantage in the game by purchasing a virtual currency or other premium features. The game publishing companies do no longer need a high amount of downloads to achieve high revenues. Competition between game publishing companies for new users mostly takes place at a download price of zero [7]. Today revenue of digital mobile games is driven by inapp-purchases. An in-app-purchase is a purchase that is done inside the application to use special premium features. In July $201699 \%$ of the top 200 applications by revenue in the Apple App-Store offered in-app- purchases [7].

Users of mobile gaming applications often repeat tasks inside the game. The game starts a trigger, which leads to an action of the player. The game rewards the player for completing the action. This reward can be invested by the player so that he can have an advantage in the next run. Eyal called these four stages a core loop[9]. 
Mobile gaming applications sometimes take advantage of dark gaming patterns and behavioral economic theories to motivate players to buy premium features inside the game via in-app-purchases [2] [3] [4] [8] [9]. The focus of this paper is the role of instability in virtual economies. The over expansion of a virtual hero's traits leads to instability of a game's virtual economy.

Players run through a loop while playing a mobile gaming application[9]. Game publishing companies can set up a virtual economy inside a game in a way so that people have to run through this loop more often or spend more money. If players have to run through this loop too fast and too often to compete with others they might stop playing the game. Sunk cost fallacy might be a reason why they continue playing even though they don't like the game anymore [3]. This contradiction will be discussed in a case study of the mobile gaming application "War of Nations". "War of Nations" is a mobile gaming application that was successful in many different countries some years ago. Over the years the game has lost it's top position within the apple app-store. This paper will have a closer look at why the decline of the ranking for this particular mobile gaming application happened.

After a literatur review, the research question will be introduced. To answer the research question, a theoretical framework and the role of commanders inside the game need to be discussed. The theoretical background will lead to research method and possible biases. A first view of the collected data is presented in the chapter descriptive statistics. Based on the theoretical framework and the collected data a hypothesis will be formulated and a regression analysis will verify it. In the last chapter conclusions and further research approaches will be discussed.

\section{Literature review}

Zagal, Björk and Lewis published a paper on "Dark Patterns in Game Design" [8]. In their paper, the authors deal with dark game patterns. Dark game patterns are defined by the authors as a negative experience for the player, which is intentionally generated by the respective game company. Zagal, Björk and Lewis define monetary dark game patterns in a way that any in-game purchases a player regrets are caused by dark gaming patterns. In dark gaming patterns the player loses track of how much money he has already spent. If the player is not sure about what exactly he is buying or how much he has to buy to reach his respective target within the game, then there may also be a dark game pattern. Games are no longer just products. They changed to services. People use games over a longer period of time and the game publishers keep updating the game. In 2014 Oscar Clark has discussed this phenomenon in his book "Games as a service. How free to play can make better games".

There is research about similarity between real and virtual worlds. The field of human- computer interaction is discussed in the book "Online Worlds: Convergence of the Real and the Virtual" by Bainbridge et al.

In 2007 Friedman et al. published a scientific paper about sunk cost fallacy. Their paper is about a laboratory experiment. In their experiment they used a computer game that confronted players with different variations of sunk cost.

In 2014 Dimitar Draganov has published his book "Freemium mobile games - Design \& Monetization". In his book he discussed how game design can keep people interacting with a digital game over a longer period of time and how to convert players of a game into paying customers.

B. F. Skinner has laid the behavioral economic foundation for variable payoffs with his research into operand conditioning. His research indicates that a variable payoff is a stronger motivator for people than a predictable payoff. B. F. Skinner was known for his experimental setup in a box, which he used to research operand conditioning. This experimental setup has been included in literature under the term Skinnerbox. Variable earnings are common in digital games. This can be a motivation for playing a game longer.

$\mathrm{N}$. Eyal has discussed the concept of the core loop in his book "Hooked". Core loops are composed of the four phases trigger, action, (variable) reward and investment. A player goes through these phases when he is playing a game. According to Eyal, these four phases are behavioral and can be used to allow a player to interact with a game for as long as possible.

\section{Research question}

Most of the relevant literature discusses the behavior of humans in digital games and virtual economies on a general level. Only a few studies like Friedman et al in 2007 published about specific experiments and not on a wide unspecific general level. This paper focuses on an empirical case study. The advantage of an empirical case study is that conclusions can be made not only on a specific level. The presented research in this paper is close to a field study.

The central research question is, how instability in the virtual economy of the digital mobile game "War of Nations" has affected the revenue over time.

\section{Theoretical framework}

Players often develop an emotional connection to a game. This emotional connection is not only determined by the fun a player has from playing the game. The longer a player plays a game and the more money a player spends on a digital mobile game the more rational it becomes to him to spend even more time and money on this game. The more time and money are spent on a game the higher are the player's sunk costs. Quitting a game will confront the player with the maximum amount of sunk 
costs. It is rational for a player who already is at a higher level in a digital mobile game to continue playing this game even if another game would give him a higher amount of happiness. These players consider sunk cost and have an emotional connection to the game because they build up their own account and already have achieved success in a game. The longer players play a digital mobile game the harder it becomes for them to abandon this game. Some players will continue to play even if they don't like the game any more. Their choice is between two negative payoffs. These choices are the sunk cost of abandoning the game and the cost of staying inside the game.

In behavioral economics this effect is called the sunk cost fallacy. Sunk cost fallacy is an effect that describes the choice between two options with different sunk cost. Only one option is possible to be taken. It often seems to be rational to people to use the option with higher sunk cost. Even if the option with smaller sunk cost gives the person a higher value. Staying inside the game will increase the sunk cost for the player. The incentive to stay inside the game increases the longer a player already plays the game or the more money he already had spent.

If a player is confronted with loss in value of his account there is a huge stimulus that a player, who already spent a longer time playing a game or already spent money on a game, will increase his ambitions inside a game. Loss in valuation of a gaming account can occure because of events like instability inside a virtual economy. Instability in a virtual economy can happen in many different ways. One example is the announcement of a new very strong hero. If the new hero is a lot stronger compared to previous heroes, he makes these older heroes completely worthless. Players will have to get this new hero as fast as possible to have an advantage over other players. In the medium run all players will need this new hero to stay competitive inside the game.

The stimulus of loss happens because of sunk cost bias. Usually an additional gained unit gives the user a positive increase in utility valuation. The marginal utility unit is positive, but decreases as shown in the following figure. Smaller gains are relatively higher valuated than larger gains. A loss in utility should be the same, but it isn't. There is an asymmetry around the neutral point. People in general overvalue losses compared to gains. This is shown by the red line in the figure 1 .

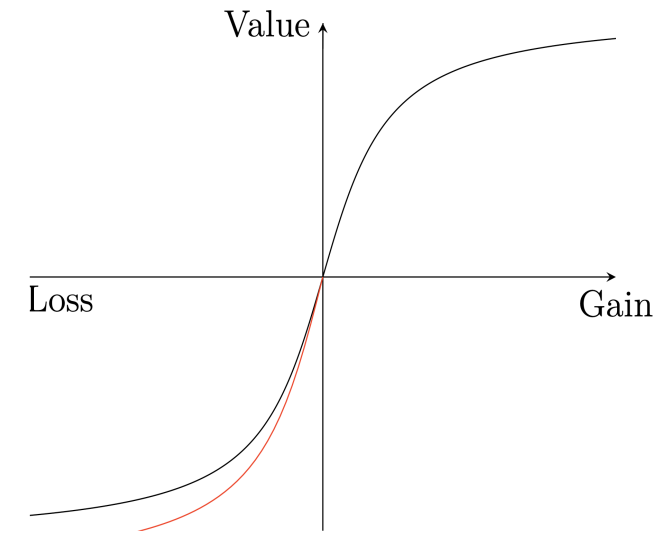

Figure 1. Sunk cost bias (own figure based on Kahneman, Knetsch and Thaler)

Transferring the sunk cost bias in the context of sunk cost fallacy in mobile digital games, this leads to the result that a small amount of instability inside a virtual economy is needed to increase profit. A small instability inside the virtual economy of a digital mobile game confronts players with a disproportionately amount of loss. Players loss aversion can increase profits of game publishing companies. The higher the instability inside of a virtual economy the higher will be the loss the players are confronted with. By increasing the time spent on the game the players are confronted with higher sunk costs. The game design only has to trigger the loss aversion of the players. Loss aversion leads to a phenomenon called "sunk cost bias". A loss of the players achievements can happen due to instability in virtual economies. The success a player has achieved is reduced compared to the moment the instability started. The prospect of loss is a more powerful motivator for peoples behavior than the prospect of a gain. The overvaluation of loss can stop people from quitting the game. This can lead to sunk cost fallacy. People have the choice between quitting the game or spending even more time and / or money on a game. This can conduct to higher profits for companies. It becomes profitable to confront players with loss. Instability in a virtual economy is a way to do so. Players can continue to play or quit the game. Quitting the game means the maximum amount of sunk costs to the player. Players will avoid this option as long as possible. Some players will even continue to play, while not having fun, but to avoid negative emotions. This is a social undesirable status.

\section{The role of commanders inside the game}

War of Nations was first released in June 2013. In this mobile digital game players compete against each other or in teams. Players build up an army and bases. There are 
different units. Each kind of unit has a certain unit value. Buildings are inside bases and can be levelled up to a maximum level. Players hit other players' bases with their army. They try to win temporary events inside the game and earn rewards. These rewards are usually set up to increase the players' or teams' strength and give the player or the team an advantage in future events. Players and teams also compete in battlepoints. Battlepoints are the sum of total unit value destroyed by a player or his team.

Commanders are virtual heroes and the most important type of boost for the player's army. Each commander has traits for attack, damage and leadership. The stronger the commander's traits, the stronger is the player's army while attacking or defending. There has been a significant increase in the strength of available commanders in the mobile digital game "War of Nations" over the last years. Players need to collect experience points for each commander. This is how commanders can reach higher levels. Commanders of the same kind can be merged. The stronger the two merged commanders are, the stronger the new commander becomes. The level of the commander increases by merging commanders up to a maximum level. In the relevant literature this mechanism is called "complete gacha". This means that players actually need a specific number of commanders of the same kind to get one really strong commander that can reach the maximum level. Players need to earn these commanders by winning events or making in-app-purchases.

In this paper, the average sum of commander traits per quarter is used as a proxy variable for instability inside the game's virtual economy.

\section{Research method and possible biases}

The company behind "War of Nations" was asked on email and many other ways to support this academic research by publishing statistics about commander traits. They did not respond. To get an estimate about the increase of commander traits social media and game specific forums have been looked up for information about commander traits[12] [13] [14] [15]. The earliest data about commander traits have been found from the 2nd quarter of the year 2014. This research focuses on the time from 2 nd quarter of 2014 until the 4th quarter of the year 2018 .

Based on the data about each commander's traits average sums per quarter from the $2^{\text {nd }}$ quarter of 2013 until 4th quarter 2018 have been calculated. The ranking is an average out of all available countries from appfigures $^{2}$ in the top grossing gaming categories for

\footnotetext{
${ }^{2}$ List of all available countries on appfigures with data for "War of Nations": Angola, Arabic Emirates, Armenia, Australia, Austria, Azerbaijan, Belgium, Bermuda, Botswana, Brazil, Brunei Darussalam, Bulgaria, Cambodia, Canada, Cayman Islands, Colombia, Costa
}

iPhone and ipad. For each quarter an average rank has been calculated. There is no public data available on how large each countries market for "War of Nations" actually is. Therefore no weighting for given countries has been done. The average sum of commander traits per quarter has been calculated based on the data from social media and forums[12] [13] [14] [15]. The rankings are indicators for the popularity and the revenue of the game. The change in commander traits compared to the previous quarter is an indicator for the loss aversion the game tries to confront the players with. The stronger the current commander gets, the weaker older commanders become.

There are unobserved variables in the following regression analysis. There is no public data available on when a sale happened inside the game. This might have an impact on the game's top grossing ranking. Sales within other games might have a negative influence on the game's top grossing ranking.

Other economic factors are not assumed in the model, because the following model is using global average data for the game's top grossing ranking. Macroeconomic variables should be added to the model in case that the ranking for different countries is compared. This is not the case in this study.

Based on these information the following research has been done. Player boosts also affect commander traits and since stronger players are more likely to post information of their commanders there might be an upwards bias in the data.

\section{Descriptive statistics}

The collected data are presented in the following figure. The left $y$-axis of the figure 2 shows the ranking in the category "Gaming Top Grossing", which is an indicator for the revenue relative to all other competitors. The right $\mathrm{y}$-axis of the figure 2 shows the commander traits in $100 \mathrm{k}$ units. Data on commander traits are available from 2 nd quarter of 2014 . On the $x$-axis the quarters from the $2^{\text {nd }}$ quarter of 2013 until the 4th quarter of 2018 are shown. Each point in figure 2 gives the

Rica, Croatia, Cyprus, Czech Republic, Denmark, Dominican Republic, Ecuador, Egypt, Finland, France, Germany, Ghana, Greece, Honduras, Hong Kong, Hungary, India, Indonesia, Ireland, Israel, Italy, Jamaica, Kazakhstan, Kuwait, Lao Peoples Democratic Republic, Latvia, Lebanon, Luxembourg, Malaysia, Malta, Mexico, Namibia, Netherlands, New Zealand, Nigeria, Norway, Pakistan, Panama, Papua New Guinea, Peru, Philippines, Poland, Portugal, Qatar, Romania, Russia, Saudi Arabia, Singapore, Slovakia, Solomon Islands, South Africa, Spain, Switzerland, Thailand, Trinidad and Tobago, Tunisia, Turkey, Tuvalu, Ukraine, United Kingdom, United States, Venezuela, Vietnam 
average per quarter for the iphone / ipad category or the average sum of commander traits per quarter.

The introduction and growth phase can be seen from the $2^{\text {nd }}$ to $3^{\text {rd }}$ quarter of 2013 . From the $4^{\text {th }}$ quarter of 2013 until $2^{\text {nd }}$ quarter of 2014 the maturity phase of the product life cycle happened. After this the decline happened. The decline stabilized, when the average sum of commander traits started to increase. The average sum of commander traits is the proxy variable for the game's virtual economy. In the $2^{\text {nd }}$ quarter of 2016 a big decline in the game's ranking occured. The big decline happened nearly a year after the instability started. After the huge drop the ranking stabilized between rank 350 and 450 .

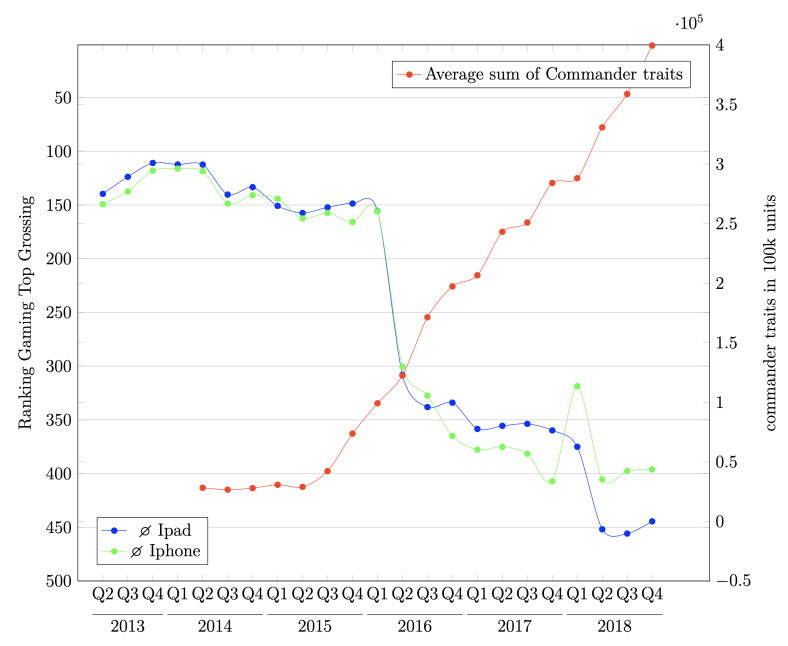

Figure 2. War of Nations: Average Ranking of all available countries and average sum of commander traits per quarter (own figure, data based on appfigures[1], social media and game forums [12] [13] [14] [15])

\section{Hypothesis}

The previously discussed leads to the hypothesis that an increase in commander traits might have happened too quick in the past of the mobile gaming application "War of Nations". A short term instability might lead to an increase in revenue in short term, but a long term instability of a virtual economy leads to a decrease of revenue.

Players of mobile digital games tend to be happy about the introduction of new contend to the game. This usually leads to a positive impact of new content to revenue. The positive effect of instability in the virtual economy of the mobile gaming application "War of Nations" happened between the $3^{\text {rd }}$ quarter of 2015 and the $1^{\text {st }}$ quarter of 2016. Although there was a positive impact of instability in the virtual economy a better ranking couldn't be achieved, but the ranking stabilized. From the $2^{\text {nd }}$ quarter of 2016 the negative effect of instability in the game's virtual economy took over and the ranking started to decrease.

The players had to go through the game's core loop more often than they felt comfortable with. This means that even though there is a sunk cost fallacy over time players will quit the game, because the new virtual commanders are a lot stronger than previous virtual commanders. The new game deciding content deflated the subjective value of older virtual commanders. This process should be able to be seen in a decrease of the ranking in the category "Gaming Top Grossing".

\section{Regression analysis}

The question that has to be answered is, how the impact of the average sum of commander traits per quarter to the average ranking of the mobile gaming application "War of Nations" per quarter in the top grossing category changed over time. There will be a significant change in ranking, which happened once in the data. To model this one time change, the functional form is assumed to be like this:

Ranking $_{i}=\beta_{0}+\beta_{1} \cdot$ Commander $+\beta_{2} \cdot$ Commanderhigh $+\mathcal{E}_{i}$

Ranking is the dependent variable. Commander is an independent variable for the average sum of commander traits per quarter. Commanderhigh is a dummy variable that equals 1 , if the average sum of commander traits is above 100.000 . Commanderhigh is the variable that gives further information about the one time effect of a high average sum of commander traits on the ranking. The value of 100.000 for high commander traits as a dummy variable has been chosen, because this value gives the highest $\mathrm{R}^{2}$.

If there is a negative effect of commander traits towards the ranking of the mobile gaming application "War of Nations" in the top grossing category the independent variable for Commander and Commanderhigh need to be positive. Both have to be statistically significant. The dummy variable Commanderhigh shifts the intercept and gives information about when the effect of the average sum of commander traits per quarter has an even stronger impact on the ranking. To avoid serial correlation generalized least squares has been used. The regression result for the top grossing iPhone category is the following:

Ranking $_{i}=126.9367+0.0004188 \cdot$ Commander $+136.4343 \cdot$ Commanderhigh $+\mathcal{E}$

The $\mathrm{R}^{2}$ is 95.03 and the adjusted $\mathrm{R}^{2}$ is 94.75 . The $\mathrm{P}$ values are 0 for Commander and Commanderhigh. Given a 5 percent significance level, this means that there is a 
statistical significant effect between these two independent variables and the dependent ranking variable.

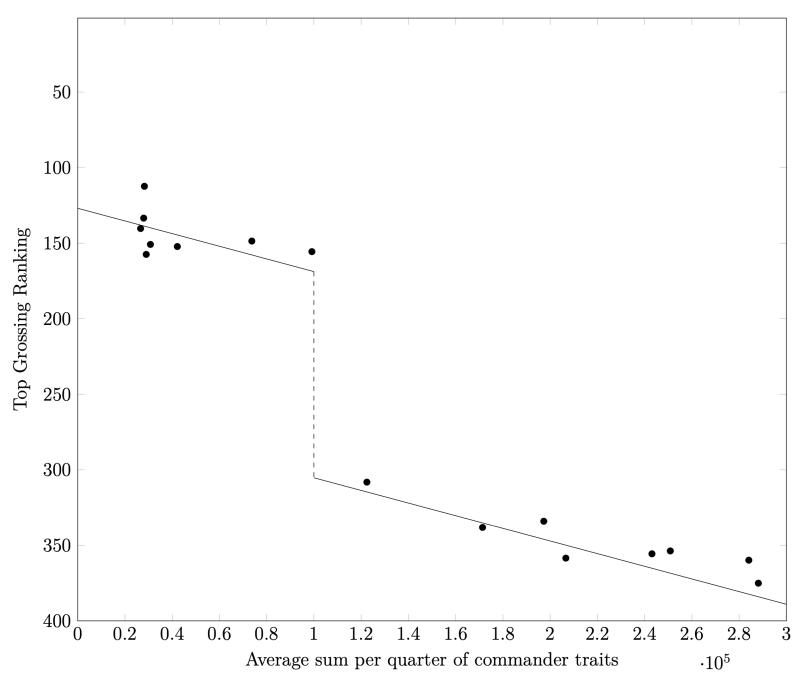

Figure 3. Regression plot quarter (own figure, data based on appfigures[1], social media and game forums [12] [13] [14] [15])

The figure 3 shows the regression function using generalized least squares. The moment the proxy variable for the game's instability in the virtual economy rises too high the ranking drops. In this functional form the slope before and after the drop in ranking is the same.

If the average sum of commander traits increase by one unit and all other factors stay constant, the ranking in the category "Gaming Top Grossing" increases by 0.0004188 . This means that the number of the ranking gets higher. The game gets a lower ranking in the respective stores. When the average sum of commander traits per quarter reaches 100.000 , the intercept increases by 136.4343 , because of the dummy variable commanderhigh.

The hypothesis can be confirmed. Players of the mobile digital game "War of Nations" stayed inside the game's core loop for three quarters after the instability inside the virtual economy occured. Temporary positive effects can be interpreted. The downwards trend of the gaming application could be slowed down for three months. In 2016 the negative effect of instability in the virtual economy started and the game's ranking dropped. The drop can be seen in the dummy variable commanderhigh.

After three quarters players anticipated the introduction of new stronger commanders inside the game and the devaluation of previous game deciding content. The change in players anticipation about new content is the central reason why the drop in ranking occured.

\section{Conclusion and further research approaches}

Some mobile digital games are designed to strengthen effects of loss aversion and sunk cost fallacy. An exploitation of these effects can happen by a high instability inside a virtual economy. This instability can last for a short, medium or a long time period.

A high instability in a virtual economy is a way to exploit players. The ranking stabilized during the first quarters after the introduction of the instability inside the virtual economy. If instability in a virtual economy happens for a longer period of time or increases too high the game looses it's players. Players are the customers of game publisher companies. Customers might get excited about new content in the first moments, but adding more and stronger game deciding contents will not increase customer satisfaction in medium or long run. In the medium or long run, there is no other way for players to react to things like a high instability in virtual economies than quitting the game. This means the maximum amount of sunk costs for the players. A socially undesirable situation for some players can be the consequence, because some players will avoid to quit playing as long as possible. For some players this can mean to continue playing without satisfaction.

A small amount of instability inside a game's virtual economy might be necessary to keep a game interesting for new players. Mobile digital games usually reward staying in the game for a long time. The longer players continue to play the stronger their accounts get. This can make it hard for players to compete inside a game with players who play a game for a longer period of time. A small increase in commander traits and the overall virtual economy can help to make old rewards useless after some time. In this way a small increase compared to previous time periods inside a virtual economy can help to balance a game.

The presented model in this study has limits. The effect of the commander variable might be higher in one country compared to other countries. Unobserved variables might have an impact on the ranking, too.

Instability in virtual economies is a field that deserves further research. There is a research gap at the moment. Game publishing companies can take advantage of their customers by confronting them with loss. One important question that needs more researchers' attention is how huge the socially undesirable effect for customers of mobile digital games is.

At the moment publishers of mobile digital games are not faced with many regulations. There are discussions about regulating lootboxes. In addition to the discussed topics in this papers there are a lot of asymmetric information in the business of mobile digital games. These asymmetric information are worth a further discussion, too.

Corporate social responsibility can be a solution to increase customer satisfaction and reduce socially undesirable situations for customers. A code of conduct 
for human game design can be a step in the right direction to avoid a socially undesirable status for players. A limitation of instability in virtual economies of mobile digital games can be a part of such a code of conduct. An exploitation of player's loss aversion is a socially undesireable situation. The reduction of this exploitation is recommended.

\section{References}

[1] Appfigures. 2018. War of Nations - PVP Strategy, https://appfigures.com/reports/ ranking

[2] B.F. Skinner. 1959. Cumulative Record, Meredith Corporation, Definitive Edition.

[3] D. Friedman, K. Pommerenke, R. Lukose, G. Milam, B. A. Huberman. 2007. Searching for the sunk cost fallacy. Economic Science Association, DOI 10.1007/s10683-0069134-0

[4] D. Kahneman, J. L. Knetsch, R. H. Thaler. 1991. Anomalies The Endowment Effect, Loss Aversion, and Status Quo Bias. Journal of Economic Perspectives, Volume 5, Number1

[5] D. Draganov. 2014. Freemium Mobile Gaming - Design \& Monetization. CreateSpace Independent Publishing Platform. ISBN: 1512322172

[6] G. Mangan, S. Sophister. 2016. Pricing's Next Top Model: A Game Theoretic Analysis of 'Freemium' Pricing. The Student Economic Review 2016. https://www.tcd.ie/Economics/assets/pdf/SER/2016/Manga n_Pricings_Next_Top_Model.pdf

[7] G. Spencer. 2016. Exploring the App Store's Top Grossing Chart. MacStories, https://www.macstories.net/stories/exploring-the- appstores-top-grossing-chart

[8] J. Zagal, S. Björk, C. Lewis. 2013. Dark Patterns in the Design of Games, Foundations of Digital Games

[9] N. Eyal. 2014. Hooked, Redline publishing company, Volume 3, ISBN: 978-3-86881-536-8

[10] O. Clark. 2014. Games as a service - How free to play design can make better games. Focal Press Taylor and Francis group. ISBN: 978-0-415-73250-5

[11] T.Wijman. 2019. The global games market will generate $\$ 152.1$ billion in 2019 as the U.S. overtakes china as the biggest market. https://newzoo.com/insights/articles/theglobal-games-market-will-generate-152-1-billion-in-2019as-the-u-s-overtakes-china-as-the-biggest-market

[12] "War of Nations" Facebook group. 2018. https://www.facebook.com/groups/195959667240038/

[13] "War of nations tips tricks and forum" Facebook group. 2018.

https://www.facebook.com/groups/246277232203378/

[14] "War of Nations" Facebook page. 2018. https://www.facebook.com/warofnations/

[15] "War of Nations Forum". 2018. https://forums.popreach.com/forumdisplay.php?f=577

[16] W. Sims Bainbridge et al. 2008. Online Worlds: Convergence of the Real and the Virtual. Springer-Verlag, ISBN 978-1-84882-824-7 